\title{
Acute Kidney Injury Biomarkers: Are We Ready for the Biomarker Curve?
}

\author{
Claudio Ronco \\ Department of Medicine, University of Padua, San Bortolo Hospital, Vicenza, Italy
}

Acute kidney injury (AKI) is common in critically ill patients and carries severe complications and a high risk of mortality [1]. Consequently, all preventive and protective strategies should be implemented to mitigate the effects of the syndrome. Early recognition of patients at risk due to identifiable exposures or increased susceptibility is a cornerstone of AKI management. Several lines of evidence suggest that markers of acute tubular stress/damage such as TIMP-2 and IGFBP7 (cell cycle arrest biomarkers identified as Nephrocheck [NC]) are useful in the risk assessment for AKI [2,3]. NC has a very high predictive ability to identify patients likely to develop stage 2/3 AKI within $12 \mathrm{~h}$ [4]. In a recent Acute Disease Quality Initiative meeting, a consensus was reached on the possibility to include biomarker results in the definition/classification of AKI utilizing both functional and damage criteria for the diagnosis [5]. Today diagnosis of the syndrome still relies on creatinine and urine output but they only meet the criteria for AKI once kidney dysfunction becomes manifest. There is evidence however that $\mathrm{NC}$ and other biomarkers may help to identify patients with tubular damage that may or may not evolve into a clinically manifest syndrome [6, 7]. Furthermore, biomarkers can help to identify patients with worse long-term outcomes [8, 9]. In spite of an important body of literature, there is still a remarkable resistance to the adoption of AKI biomarkers in clinical routine [10]. Skeptical and nihilistic physicians continue to ask for more evidence while enthusiastic groups that would love to adopt biomarkers are frustrated by repeated denials justified by the cost of these diagnostic tools $[2,11]$. The Sapphire investigators and others demonstrated that NC predicts AKI stage 2-3 within $12 \mathrm{~h}$ before serum creatinine rise $[2,12]$. Other studies suggest that new biomarkers significantly add diagnostic and prognostic certainty and enable improved AKI risk assessment in high-risk patients [13]. Although they do not uncover the predominant type of injury (ischemic, toxic, inflammatory) or the specific site of damage (vascular, proximal/distal tubules, etc.), their use appears today definitely justified $[2,14]$.

The nature of critical illness is however such that patients in the intensive-care unit (ICU) are subject to a subsequent series of insults and their risk to develop AKI may be modified within hours by new exposures. Multiple insults may derive from nephrotoxic drugs, sepsis 
and infection episodes, emergency surgery, contrast media for imaging techniques. In such conditions, the risk of AKI could be anticipated with multiple serial NC determinations. The paper by McCullough et al., published in this issue [15], evaluates the utility of NC serial measurements in a retrospective post hoc analysis of the Sapphire ICU patients' cohort and suggests that serial NC ([TIMP-2] $\times$ [IGFBP7]) measurements can be useful to predict stage 2-3 AKI over the course of the first 7 days of ICU stay. Although the authors acknowledge the limitations of the study (retrospective design and small sample size), the analysis adds important information on the utility of biomarkers of tubular injury in predicting AKI burden throughout the ICU stay. This study may in fact lead to the development of a better hemodynamic monitoring and mitigation of additional superimposed nephrotoxic insults during critical illness. The authors demonstrated that serial measurement and interpretation of urine [TIMP-2] $\times$ [IGFBP7] in critically ill patients every $12 \mathrm{~h}$ for at least 3 samples provided an approach to predict the progressive risk of stage $2 / 3$ AKI up to 7 days in the ICU [15]. Additionally, there appeared to be value in the utilization of both the 0.3 and $2.0(\mathrm{ng} / \mathrm{mL})^{2} / 1,000$ cutoff points with persistently negative and emerging or persistently positive/strongly positive results anticipating very low or progressively higher rates of the primary end point, respectively. They conclude that serial urinary [TIMP-2] $\times$ [IGFBP7] at baseline, 12 and $24 \mathrm{~h}$ and up to 3 days are prognostic for the occurrence of stage $2 / 3 \mathrm{AKI}$ over the course of critical illness. Three consecutive negative values $\left(\leq 0.3(\mathrm{ng} / \mathrm{mL})^{2} / 1,000\right)$ are associated with a very low (13.0\%) incidence of stage $2 / 3$ AKI over the course of 7 days. Conversely, emerging or persistent, strongly positive results $\left[>2.0(\mathrm{ng} / \mathrm{mL})^{2} / 1,000\right]$ predict very high incidence rates (up to $94.4 \%$ ) of stage $2 / 3$ AKI [15].

Other authors had previously suggested that serial measurement of AKI biomarkers could be useful to better characterize the kidney risk profile of patients during their stay in the ICU [16]. Since critically ill patients present multiple potentially dangerous exposures that can result in AKI, biochemical profiles including serum creatinine and clod urea nitrogen are routinely measured daily in the ICU. Therefore, it would make sense to carry out a similar biochemical monitoring with biomarkers, once their utility for AKI prediction has been established. Data from the study of McCullough et al. [15, this issue] suggest that that predictive information gained on successive samples is somewhat cumulative for those who have not developed AKI quickly, and thus suggest the value for surveillance when the patient remains critically ill and vulnerable to AKI. After discovery and validation of cell cycle arrest biomarkers have been published in the original Sapphire trial [4], some studies have corroborated the utility of these biomarkers in triggering specific interventions [17-19]. The results reported in the post hoc Sapphire analysis introduce the question as to whether the dynamic response of the markers to new exposures could also guide ongoing management. The results of the PrevAKI randomized trial have demonstrated that [TIMP-2] $\times$ [IGFBP7] can be used to trigger a set of care processes based on KDIGO guidelines including the use of hemodynamic monitoring and inotropic therapy, resulting in reduced rates of subsequent AKI after cardiac surgery [17]. Our own studies demonstrated the utility of the application of a nephrology rapid response team triggered by a positive biomarker determination [18-20]. The authors extend the inferences from PrevAKI and suggest continued daily surveillance for the risk of AKI has clinical value, in all three relevant [TIMP-2] × [IGFBP7] test result scenarios: persistently negative, negative transforming to positive, and persistently positive/strongly positive. They describe fairly clear patterns: only few patients remain in an area of uncertainty showing the utility of repeated biomarker assessment in a sort of "curve" that resembles what is commonly done today with troponin in case of a suspected acute coronary syndrome. It is likely that in the future, interventions aimed to attenuate the progression of AKI will be triggered by serial measurements of AKI biomarkers in the ICU. Further to what has been suggested by the current study of the Sapphire investigators, multiple profiles could become 


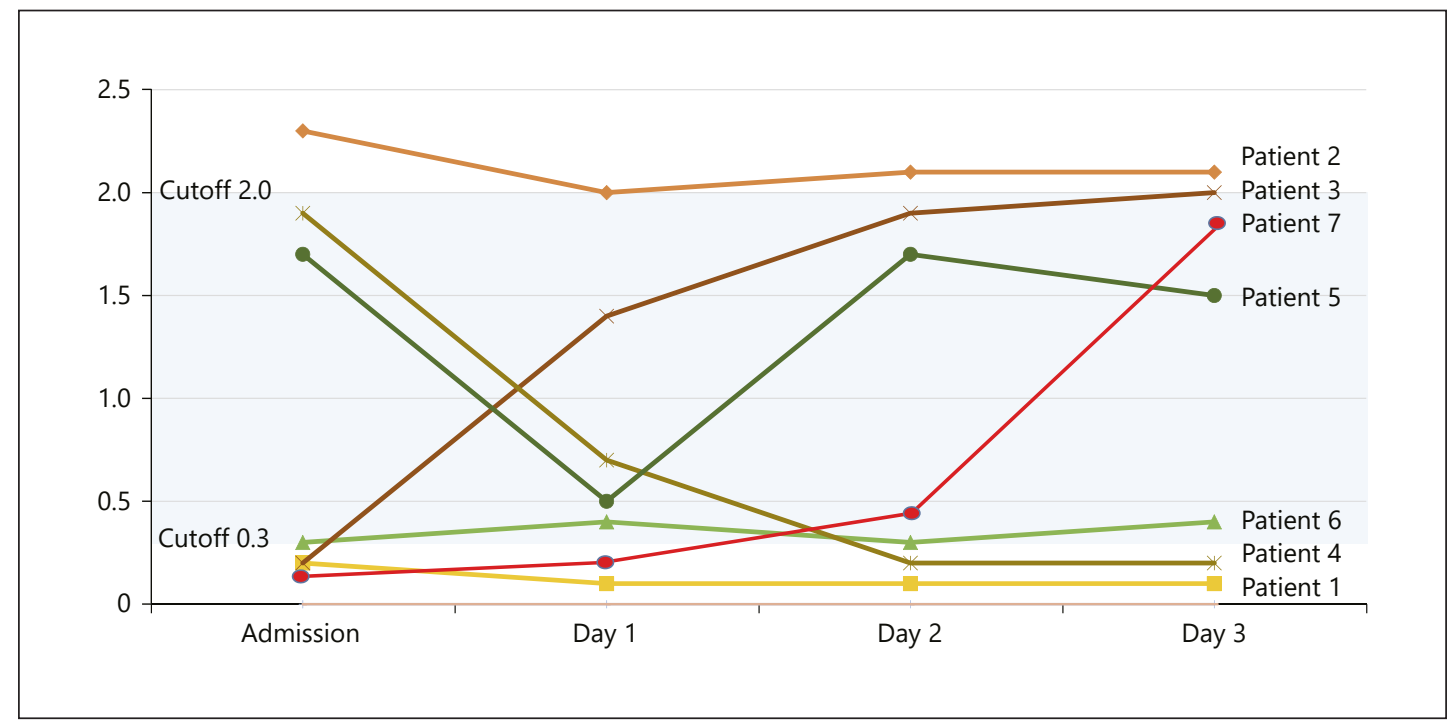

Fig. 1. Possible [TIMP-2 $\times$ IGFBP7] patterns during the first 3 days of ICU stay in critically ill patients (data drawn from real cases at the Vicenza Hospital). Patient 1: persistently negative; high negative predictive value (likely never developing AKI). Patient 2: persistently above 2.0; high positive predictive value (likely developing AKI in the first days). Patient 3: progressive increase after a negative value at admission; possible sign of early insult upon admission or postoperatively (if undergone surgery) leading to AKI before day 7 . Patient 4: progressive decrease; after a pre-admission insult, the risk of developing AKI is decreasing probably due to interventions, and on day 3 a minimum risk is observed. Patient 5: bimodal peaking; patient with pre-admission insult (surgery) who received a second insult on day 1 or 2 and peaks again with an increased risk of AKI until day 7. Patient 6: persistent value above minimum cutoff point; marginal risk to develop AKI before day 7. Patient 7: late increase; typical of a late insult leading to AKI before day 7.

available to the clinician as reported from real measurements obtained in ICU patients in Vicenza and reported in Figure 1.

As in these examples, each profile will describe in the future a specific pattern of AKI suggesting how to modulate interventions and changes in management. With the routine use of biomarkers we will have an enormous amount of data useful to design specific algorithms with the aid of artificial intelligence, to guide our practice. The remaining question is: are we ready to adopt an AKI biomarker curve in a world that is still reluctant to utilize biomarkers at all? The evolution of science cannot be arrested. We will see AKI biomarker curves used for AKI risk assessment and probably diagnosis in the coming years.

\section{References}

1 Hoste EA, Bagshaw SM, Bellomo R, Cely CM, Colman R, Cruz DN, et al. Epidemiology of acute kidney injury in critically ill patients: the multinational AKI-EPI study. Intensive Care Med. 2015 Aug;41(8):1411-23.

2 Kashani K, Cheungpasitporn W, Ronco C. Biomarkers of acute kidney injury: the pathway from discovery to clinical adoption. Clin Chem Lab Med. 2017 Jul 26;55(8):1074-89.

3 McCullough PA, Bouchard J, Waikar SS, Siew ED, Endre ZH, Goldstein SL, et al. Implementation of novel biomarkers in the diagnosis, prognosis, and management of acute kidney injury: executive summary from the tenth consensus conference of the Acute Dialysis Quality Initiative (ADQI). Contrib Nephrol. 2013;182:5-12.

4 Kashani K, Al-Khafaji A, Ardiles T, Artigas A, Bagshaw SM, Bell M, et al. Discovery and validation of cell cycle arrest biomarkers in human acute kidney injury. Crit Care. 2013 Feb;17(1):R25.

5 Murray PT, Mehta RL, Shaw A, Ronco C, Endre Z, Kellum JA, et al.; ADQI 10 workgroup. Potential use of biomarkers in acute kidney injury: report and summary of recommendations from the 10th Acute Dialysis Quality Initiative consensus conference. Kidney Int. 2014 Mar;85(3):513-21. 
6 Ronco C. Acute kidney injury: from clinical to molecular diagnosis. Crit Care. 2016 Jul;20(1):201.

7 Hoste EA, McCullough PA, Kashani K, Chawla LS, Joannidis M, Shaw AD, et al.; Sapphire Investigators. Derivation and validation of cutoffs for clinical use of cell cycle arrest biomarkers. Nephrol Dial Transplant. 2014 Nov;29(11):2054-61.

8 Koyner JL, Shaw AD, Chawla LS, Hoste EA, Bihorac A, Kashani K, et al.; Sapphire Investigators. Tissue Inhibitor Metalloproteinase-2 (TIMP-2)·IGF-Binding Protein-7 (IGFBP7) Levels Are Associated with Adverse LongTerm Outcomes in Patients with AKI. J Am Soc Nephrol. 2015 Jul;26(7):1747-54.

9 Xie Y, Ankawi G, Yang B, Garzotto F, Passannante A, Breglia A, et al. Tissue inhibitor metalloproteinase-2 (TIMP-2) - IGF-binding protein-7 (IGFBP7) levels are associated with adverse outcomes in patients in the intensive care unit with acute kidney injury. Kidney Int. 2019 Jun;95(6):1486-93.

10 Fan W, Ankawi G, Zhang J, Digvijay K, Giavarina D, Yin Y, Ronco C. Current understanding and future directions in the application of TIMP-2 and IGFBP7 in AKI clinical practice. Clin Chem Lab Med. 2019 Apr 24;57(5):56776.

11 Levante C, Ferrari F, Manenti C, Husain-Syed F, Scarpa M, Hinna Danesi T, et al. Routine adoption of TIMP2 and IGFBP7 biomarkers in cardiac surgery for early identification of acute kidney injury. Int J Artif Organs. 2017 Nov; 40(12):714-8.

12 Ostermann M, McCullough PA, Forni LG, Bagshaw SM, Joannidis M, Shi J, et al.; all SAPPHIRE Investigators. Kinetics of Urinary Cell Cycle Arrest Markers for Acute Kidney Injury Following Exposure to Potential Renal Insults. Crit Care Med. 2018 Mar;46(3):375-83.

13 Di Leo L, Nalesso F, Garzotto F, Xie Y, Yang B, Virzì GM, et al. Predicting Acute Kidney Injury in Intensive Care Unit Patients: The Role of Tissue Inhibitor of Metalloproteinases-2 and Insulin-Like Growth Factor-Binding Protein-7 Biomarkers. Blood Purif. 2018;45(1-3):270-7.

14 Bihorac A, Chawla LS, Shaw AD, Al-Khafaji A, Davison DL, Demuth GE, et al. Validation of cell-cycle arrest biomarkers for acute kidney injury using clinical adjudication. Am J Respir Crit Care Med. 2014 Apr;189(8): 932-9.

15 McCullough P et al for the Sapphire investigators. Serial urinary tissue inhibitor of metalloproteinase-2 and insulin-like growth factor-binding protein 7 and the prognosis for acute kidney injury over the course of critical illness. Cardiorenal Med. 2019; DOI: 10.1159/000502837.

16 Cummings JJ, Shaw AD, Shi J, Lopez MG, O’Neal JB, Billings FT. Intraoperative prediction of cardiac surgeryassociated acute kidney injury using urinary biomarkers of cell cycle arrest. J Thorac Cardiovasc Surg. 2019 Apr;157(4):1545-53.

17 Meersch M, Schmidt C, Hoffmeier A, Van Aken H, Wempe C, Gerss J, et al. Prevention of cardiac surgery-associated AKI by implementing the KDIGO guidelines in high risk patients identified by biomarkers: the PrevAKI randomized controlled trial. Intensive Care Med. 2017 Nov;43(11):1551-61.

18 Ronco C, Rizo-Topete L, Serrano-Soto M, Kashani K. Pro: prevention of acute kidney injury: time for teamwork and new biomarkers. Nephrol Dial Transplant. 2017 Mar 1;32(3):408-13.

19 Rizo-Topete LM, Rosner MH, Ronco C. Acute Kidney Injury Risk Assessment and the Nephrology Rapid Response Team. Blood Purif. 2017;43(1-3):82-8.

20 Koyner JL, Zarbock A, Basu RK, Ronco C. The impact of biomarkers of acute kidney injury on individual patient care. Nephrol Dial Transplant. Forthcoming 2019. 